\title{
Tigecycline-based versus sulbactam-based treatment for pneumonia involving multidrug-resistant Acinetobacter calcoaceticus-Acinetobacter baumannii complex
}

\author{
Jung-Jr Ye', Huang-Shen Lin², Chun-Fu Yeh', Yen-Mu Wu', Po-Yen Huang ', Chien-Chang Yang ${ }^{1}$, \\ Ching-Tai Huang ${ }^{1}$ and Ming-Hsun Lee ${ }^{1^{*}}$
}

\begin{abstract}
Background: The treatment options for pneumonia involving multidrug-resistant Acinetobacter calcoaceticusAcinetobacter baumannii (MDR Acb) complex are limited, and the optimal treatment has not been established.

Methods: To compare the efficacy of tigecycline-based with sulbactam (or ampicillin/sulbactam)-based therapy for pneumonia involving MDR Acb complex, we conducted a retrospective study comparing 84 tigecycline-treated adult patients during the period August 2007 to March 2010 with 84 sulbactam or ampicillin/sulbactam-treated adult patients during the period September 2004 to July 2007. Both groups had the matched Acute Physiology and Chronic Health Evaluation (APACHE) II score and received treatment for at least 7 days.
\end{abstract}

Results: The mean APACHE II score was 20.1 for both groups. More patients in sulbactam group had ventilator use $(89.3 \%$ versus $69.0 \%$ ), bilateral pneumonia (79.8 \% versus $60.7 \%$ ) and combination therapy ( $84.5 \%$ versus $53.6 \%)$, particularly with carbapenems $(71.4 \%$ versus $6.0 \%$ ), while more patients in tigecycline group had delayed treatment $(41.7 \%$ versus $26.2 \%)(P<0.05)$. At the end of treatment, more patients in sulbactam group had airway MDR Acb complex eradication ( $63.5 \%$ versus $33.3 \%, P<0.05$ ). The clinical resolution rate was $66.7 \%$ for both groups. The mortality rate during treatment was $17.9 \%$ in sulbactam group, and $25.0 \%$ in tigecycline group $(P=0.259)$. The multivariate analysis showed that bilateral pneumonia was the only independent predictor for mortality during treatment (adjusted odds ratio, 2.717; $95 \%$ confidence interval, 1.015 to 7.272).

Conclusions: Patients treated with either tigecycline-based or sulbactam-based therapy had a similar clinical outcome, but tigecycline group had a lower microbiological eradiation rate.

\section{Background}

Pneumonia involving multidrug-resistant (MDR) Acinetobacter calcoaceticus-Acinetobacter baumannii (Acb) complex usually occurs in critically ill patients and is associated with unfavorable outcomes [1-3]. For MDR Acb complex resistant to most currently available antibiotics, including $\beta$-lactams, fluoroquinolones, and aminoglycosides, there

\footnotetext{
* Correspondence: drharrylee@gmail.com

${ }^{1}$ Division of Infectious Diseases, Department of Internal Medicine, Chang Gung Memorial Hospital at Linkou, Chang Gung University College of Medicine, 5 Fu-Shin St.Gueishan 333 Taoyuan, Taiwan

Full list of author information is available at the end of the article
}

are only a few treatment options, such as tigecycline, sulbactam, and colistin $[4,5]$.

Tigecycline is a glycylcycline with in vitro activity against MDR Acb complex [6]. The comparison analysis from the U.S. Food and Drug Administration showed that tigecycline treatment had a higher mortality rate than other antimicrobials in ventilator associated pneumonia (VAP) [7]. A recent study also reported a significantly lower cure rate in clinically evaluable patients with VAP treated with tigecycline when compared to imipenem $(47.9 \%$ versus $70.1 \%)$ 
[8]. However, for pneumonia caused by MDR Acb complex resistant to carbapenems and other classes of antibiotics, off label use of tigecycline was common in clinical practice, and the clinical response rates ranged from 60 to $88 \%$ in prior studies [9-11]. Sulbactam is a $\beta$-lactamase inhibitor with antimicrobial activity against Acinetobacter species [12]. It is available alone or in combination with ampicillin, and ampicillin doesn't contribute activity or synergism against $A$. baumannii [12]. Sulbactam or ampicillin/sulbactam had clinical response rates ranging from 67 to $75 \%$ for pneumonia involving MDR A. baumannii (MDRAB) or MDR Acb complex in prior studies [13-15].

In our hospital, tigecycline was not available until August 2007. Before that, sulbactam or ampicillin/sulbactam might be the only treatment option with in vitro activity against MDR Acb complex. Thus, we conducted a retrospective study to compare the efficacy of tigecycline-based with sulbactam (or ampicillin/ sulbactam)-based treatment for pneumonia involving MDR Acb complex. With a match in the Acute Physiology and Chronic Health Evaluation (APACHE) II score for both groups, a comparison was made between tigecycline-treated adult patients during the period August 2007 to March 2010 and sulbactam (or ampicillin/sulbactam)-treated adult patients during the period September 2004 to July 2007. The clinical efficacy, outcomes and microbiological eradication were included for analyses.

\section{Methods}

\section{Setting}

Chang Gung Memorial Hospital (CGMH)-Linkou is a university-affiliated medical center providing both primary and tertiary health care in northern Taiwan. This retrospective study has been approved by institutional review boards of CGMH- Linkou (Number: 99-1478B and 100-0294B). The ethics committee granted a waiver for informed consent to be obtained.

\section{Study design, patients and treatments}

All hospitalized patients who were $\geqq 18$ years old and had pneumonia involving MDR Acb complex treated with tigecycline between August 2007 and March 2010, and sulbactam or ampicillin/sulbactam between September 2004 and July 2007, were reviewed. Each tigecycline-treated patient was matched to one sulbactam or ampicillin/sulbactam-treated patient based on identical values of APACHE II score and chart number sequence. Patients were excluded if they did not have a matched control or had a combination therapy with tigecycline and sulbactam (or ampicillin/sulbactam). Patients with initial bacteremia were also excluded since tigecycline treatment for bacteremia was controversial.
Pneumonia was diagnosed if the patient had a radiographic infiltrate that was new or progressive, along with at least two of the following clinical characteristics: new onset of fever $\left(\geqq 38{ }^{\circ} \mathrm{C}\right)$ or hypothermia $\left(<35.5{ }^{\circ} \mathrm{C}\right)$, leucocytosis (leucocyte count $>12000$ cells $/ \mathrm{mm}^{3}$ ) or leucopenia (leucocyte count $<4000$ cells $/ \mathrm{mm}^{3}$ ), decline in oxygenation (O2 saturation $<90 \%)$, and increasing amount of purulent sputum [16]. Pneumonia involving MDR Acb complex was defined as clinical evidence of pneumonia with sputum or tracheal aspirate cultures positive for MDR Acb complex from 1 week before to 3 days after the first dose of tigecycline or sulbactam or ampicillin/sulbactam. Tracheal aspirate and sputum specimens were sent for bacterial culture only if their Gram's stains showed at least 25 neutrophils and less than 10 epithelial cells per low-power field. Growth was assessed semi-quantitatively. The etiologic pathogen of pneumonia was determined if the tracheal aspirate or sputum culture had an at least moderate growth, i.e., the growth confined up to primary streaking line and $>5$ colonies in secondary streaking zone [17]. Polymicrobial pneumonia was defined as one or more additional etiologic bacterial species concurrently isolated from the respiratory tract during treatment.

All patients in tigecycline group received tigecycline for at least 7 days, with a 100-mg loading dose followed by $50 \mathrm{mg}$ administered intravenously every $12 \mathrm{~h}$. All patients in sulbactam group received intravenous sulbactam $1 \mathrm{~g}$ or ampicillin/sulbactam $3 \mathrm{~g}$ (at a ratio 2:1) every 6 or $8 \mathrm{~h}$ for at least 7 days. Dose and dosing interval were adjusted according to serum creatinine levels. Combination therapy was defined as simultaneous use of another class of antibiotics for at least 3 days. These antibiotics included carbapenems (meropenem or imipenem), fluoroquinolones (ciprofloxacin or levofloxacin), amikacin, cephalosporins (ceftazidime or cefepime), piperacillin, piperacillin-tazobactam, colistimethate, and aztreonam. Delayed treatment was defined as more than 3 days between the detection of airway MDR Acb complex isolates and the first dose of tigecycline or sulbactam or ampicillin/sulbactam.

\section{Microbiology}

Identification of Acb complex depended upon Gram staining and conventional biochemical tests [18]. Briefly, the isolates were identified as species of the genus Acinetobacter based on the following properties: aerobic, Gram-negative, nonmotile coccobacillary rods with a nonfermentative, catalase-positive and oxidase-negative reaction. Acinetobacter species with glucose-oxidizing non-haemolytic characteristics were classified as Acb complex. Antimicrobial susceptibility was determined and interpreted according to the criteria of Clinical and Laboratory Standards Institute by disk diffusion method 
[19]. Susceptibility to tigecycline was determined using disk diffusion method with Mueller-Hinton agar (BD Microbiology Systems, Cockeysville, MD) with the resistant breakpoint at $\geqq 16 \mathrm{~mm}$ and susceptible breakpoint at $\leqq 12 \mathrm{~mm}$ [20]. An isolate with full or intermediate resistance to amikacin, gentamicin, cefepime, ceftazidime, aztreonam, piperacillin, piperacillin-tazobactam, ciprofloxacin, imipenem and meropenem was defined as MDR Acb complex [21].

Cultures were collected from 1 week before the first dose of tigecycline or sulbactam (or ampicillin/sulbactam) to the discharge of patients. Pathogens, sites of growth and susceptibility testing were recorded. Microbial eradication of MDR Acb complex was defined as no growth of Acb complex or susceptibility change from MDR strains to susceptible strains in Acb complex in follow-up respiratory tract cultures before and 7 days after treatment cessation. Relapse was defined as new isolation of MDR Acb complex from the respiratory tract cultures within 2 weeks after initial eradication. Initial bacteremia was defined as bacteremia at the beginning of treatment, which meant at least one positive blood culture 1 week before to 3 days after the first dose of tigecycline or sulbactam or ampicillin/sulbactam. Bacteremia during treatment was defined as at least one positive blood culture 3 days after to the end of treatment.

\section{Demography and comorbidity}

Data on age, sex, surgery, and co-morbid illness were gathered by reviewing in-patient medical records. Comorbid illness included hepatic dysfunction of a serum total bilirubin level over $2.5 \mathrm{mg} / \mathrm{dL}$ or liver cirrhosis, renal insufficiency of a serum creatinine level above $2.0 \mathrm{mg} / \mathrm{dL}$ or requirement of dialysis, chronic pulmonary disease, heart disease, diabetes mellitus, immune compromise, and hematological or solid organ malignancy. Immune compromise was defined by at least one of the following: use of prednisone or equivalent over $20 \mathrm{mg}$ per day for at least 2 weeks, organ transplant recipient, human immunodeficiency virus infection or acquired immunodeficiency syndrome, neutropenia (absolute neutrophil count less than 500 cells/mm3), use of immunosuppressive agents, and concurrent hematological malignancy.

\section{Clinical conditions and outcomes}

Ventilator use, vital signs, and infections other than pneumonia during treatment were recorded. Defervescence was defined as normal body temperature for at least 3 days at the end of treatment. Severity of illness was assessed by a modified APACHE II score, which was recorded within $48 \mathrm{~h}$ before or after the first dose of tigecycline or sulbactam or ampicillin/sulbactam. The 30-day mortality was defined as death occurring within 30 days after treatment. The chest radiographs were evaluated by at least two investigators. A series of chest radiographs were evaluated during treatment. Clinical resolution of pneumonia at the end of treatment was defined as (1) decreased pulmonary infiltrate, and (2) survival with stationary findings on chest radiographs and defervescence. Thus, patients with persistent fever or death during treatment would be defined as clinical failure if infiltrates were stationary. Progressing infiltrates were defined as clinical failure.

\section{Statistical methods}

All statistical analyses were performed using the Statistical Package for the Social Sciences for Windows (Version 15.0; SPSS Inc., Chicago, IL, USA). Categorical variables were compared using $x^{2}$ test or Fisher exact test, as appropriate. Continuous variables were tested for normality of distributions by KolmogorovSmirnov test, and then compared by Student's $t$-test or the Mann-Whitney $U$ test, as appropriate. Odds ratios (ORs) and $95 \%$ confidence interval (CI) were calculated. The survival curve was plotted by means of the Kaplan-Meier method, and the log rank test was used to compare univariate survival distribution between tigecycline and sulbactam groups. Variables with a $P$ value $<0.1$ in univariate analysis and tigecycline use were included in a logistic regression model for multivariate analysis. All tests were two-tailed, and a $P$ value of $<0.05$ was considered significant.

\section{Results}

Patients, demography and concomitant diseases

One hundred and sixteen tigecycline-treated episodes of pneumonia involving MDR Acb complex were identified in 112 patients, while 177 sulbactam or ampicllin/ sulbactam-treated episodes were identified in 173 patients. Finally, 84 tigecycline-treated patients were enrolled and matched to 84 patients treated with sulbactam (26 patients) or ampicillin/sulbactam (58 patients). The mean APACHE II score was 20.1 for both groups. In tigecycline group, 59 (70.2 \%) and 25 (29.8\%) patients had positive MDR Acb complex cultures from tracheal aspirates and sputum, respectively. In sulbactam group, 73 (86.9\%) and 11 (13.1\%) patients had that from tracheal aspirates and sputum, respectively. There was no significant difference in age, gender, and concomitant diseases between the two groups (Table 1).

\section{Clinical conditions}

Patients in sulbactam group had more ventilator use (89.3\% versus $69.0 \%$ ) and bilateral pneumonia (79.8\% versus $60.7 \%$ ) than those in tigecycline group. There were no significant differences between these two groups in the overall rates of polymicrobial pneumonia and multisite infections. Pseudomonas aeruginosa and Methicillin-resistant Staphylococcus aureus were the most 
Table 1 The comparison analysis of demography, concomitant diseases, clinical features, and outcomes between tigecycline (TG) and sulbactam (SB) groups

\begin{tabular}{|c|c|c|c|c|}
\hline \multirow[t]{2}{*}{ Variables } & \multirow{2}{*}{$\begin{array}{l}\text { TG group }{ }^{a} \\
n=84\end{array}$} & SB group ${ }^{a}$ & \multirow[t]{2}{*}{$p$} & \multirow[t]{2}{*}{ OR $(95 \% \mathrm{Cl})$} \\
\hline & & $n=84$ & & \\
\hline \multicolumn{5}{|l|}{ Demographic parameters } \\
\hline Age, yr & $69.6(15.9)$ & $70.6(15.6)$ & 0.689 & \\
\hline Male gender & $57(67.9)$ & $58(69.0)$ & 0.868 & $0.946(0.494-1.814)$ \\
\hline \multicolumn{5}{|l|}{ Concomitant diseases } \\
\hline Hepatic dysfunction & $12(14.3)$ & $6(7.1)$ & 0.134 & $2.167(0.773-6.075)$ \\
\hline Renal insufficiency & $32(38.1)$ & $32(38.1)$ & 1.000 & $1.000(0.536-1.864)$ \\
\hline Chronic pulmonary disease & $22(26.2)$ & $20(23.8)$ & 0.722 & 1.135 (0.564-2.284) \\
\hline Heart disease & $13(15.5)$ & $7(8.3)$ & 0.153 & $2.014(0.761-5.333)$ \\
\hline Diabetes mellitus & $26(31.0)$ & $35(41.7)$ & 0.149 & $0.628(0.333-1.183)$ \\
\hline Immune compromise & $13(15.5)$ & $11(13.1)$ & 0.659 & $1.215(0.511-2.891)$ \\
\hline Malignancy & $15(17.9)$ & $20(23.8)$ & 0.342 & $0.696(0.328-1.474)$ \\
\hline Surgery & $22(26.2)$ & $15(17.9)$ & 0.193 & $1.632(0.778-3.423)$ \\
\hline \multicolumn{5}{|l|}{ Clinical conditions } \\
\hline APACHE II Score & $20.1(6.1)$ & $20.1(6.1)$ & 1.000 & \\
\hline Ventilator use & $58(69.0)$ & $75(89.3)$ & 0.001 & $0.268(0.117-0.615)$ \\
\hline Pneumonia involving bilateral lung & $51(60.7)$ & $67(79.8)$ & 0.007 & $0.392(0.197-0.781)$ \\
\hline Polymicrobial pneumonia, overall & $66(78.6)$ & $62(73.8)$ & 0.469 & $1.301(0.638-2.654)$ \\
\hline \multicolumn{5}{|l|}{ Polymicrobial pneumonia, coinfection with } \\
\hline MRSA & $26(31.0)$ & $34(40.5)$ & 0.198 & $0.659(0.349-1.245)$ \\
\hline Pseudomonas aeruginosa & $33(39.3)$ & $23(27.4)$ & 0.102 & $1.716(0.896-3.285)$ \\
\hline Klebsiella spp. ${ }^{\text {b }}$ & $12(14.3)$ & $5(6.0)$ & 0.073 & $2.633(0.884-7.840)$ \\
\hline Escherichia coli & $2(2.4)$ & $2(2.4)$ & 1.000 & $1.000(0.138-7.270)$ \\
\hline Enterobacter spp. ${ }^{c}$ & $2(2.4)$ & $2(2.4)$ & 1.000 & $1.000(0.138-7.270)$ \\
\hline Serratia marcescens & $10(11.9)$ & $1(1.2)$ & 0.005 & $11.216(1.402-89.724)$ \\
\hline Stenotrophomonas maltophilia & $6(7.1)$ & $15(17.9)$ & 0.036 & $0.354(0.130-0.962)$ \\
\hline Multisite infections, overall & $33(39.3)$ & $35(41.7)$ & 0.753 & $0.906(0.489-1.678)$ \\
\hline With urinary tract infection & $13(15.5)$ & $19(22.6)$ & 0.238 & $0.626(0.287-1.369)$ \\
\hline With catheter related infection & $2(2.4)$ & $10(11.9)$ & 0.017 & $0.180(0.038-0.851)$ \\
\hline With soft tissue and wound infection & $10(11.9)$ & $5(6.0)$ & 0.176 & $2.135(0.697-6.540)$ \\
\hline With intra-abdominal infection & $8(9.5)$ & $4(4.8)$ & 0.231 & $2.105(0.609-7.279)$ \\
\hline With invasive fungal infection ${ }^{d}$ & $12(14.3)$ & $4(4.8)$ & 0.035 & $3.333(1.029-10.799)$ \\
\hline Bacteremia during treatment & $4(4.8)$ & $0(0.0)$ & 0.121 & $9.447(0.501-178.291)$ \\
\hline With TG or SB-resistant MDR Acb complex ${ }^{\mathrm{e}}$ & $16(19.0)$ & $43(51.2)$ & $<0.0001$ & $0.224(0.112-0.448)$ \\
\hline \multicolumn{5}{|l|}{ Treatment } \\
\hline Duration, days & $14.6(5.4)$ & $16.4(7.6)$ & 0.150 & \\
\hline Combination therapy, overall & 45 (53.6) & $71(84.5)$ & $<0.0001$ & $0.211(0.102-0.439)$ \\
\hline With cephalosporins & $20(23.8)$ & $8(9.5)$ & 0.013 & $2.969(1.226-7.192)$ \\
\hline With colistin & $12(14.3)$ & $0(0.0)$ & $<0.0001$ & $29.138(1.695-500.773)$ \\
\hline With carbapenems & $5(6.0)$ & $60(71.4)$ & $<0.0001$ & $0.025(0.009-0.070)$ \\
\hline With aminoglycosides & $7(8.3)$ & $1(1.2)$ & 0.064 & $7.545(0.907-62.744)$ \\
\hline With fluoroquinolones & $12(14.3)$ & $4(4.8)$ & 0.035 & $3.333(1.029-10.799)$ \\
\hline Delayed treatment & $35(41.7)$ & $22(26.2)$ & 0.034 & $2.013(1.049-3.863)$ \\
\hline
\end{tabular}


Table 1 The comparison analysis of demography, concomitant diseases, clinical features, and outcomes between tigecycline (TG) and sulbactam (SB) groups (Continued)

\begin{tabular}{|c|c|c|c|c|}
\hline \multicolumn{5}{|l|}{ Outcomes } \\
\hline Airway eradication of MDR Acb complex without relapse ${ }^{f}$ & $26(33.3)$ & $47(63.5)$ & $<0.0001$ & $0.287(0.147-0.560)$ \\
\hline Defervescence & $54(64.3)$ & $76(90.5)$ & $<0.0001$ & $0.189(0.081-0.445)$ \\
\hline \multicolumn{5}{|l|}{ Image study of lung } \\
\hline Improvement & $37(44.0)$ & $39(46.4)$ & 0.757 & $0.908(0.495-1.668)$ \\
\hline Stationary & $32(38.1)$ & $22(26.2)$ & 0.099 & $1.734(0.900-3.342)$ \\
\hline Deterioration & $15(17.9)$ & $23(27.4)$ & 0.140 & $0.577(0.276-1.204)$ \\
\hline Clinical resolution of pneumonia & $56(66.7)$ & $56(66.7)$ & 1.000 & $1.000(0.526-1.899)$ \\
\hline Mortality during treatment & $21(25.0)$ & $15(17.9)$ & 0.259 & $1.533(0.728-3.231)$ \\
\hline 30-day mortality & $28(33.3)$ & $25(29.8)$ & 0.618 & $1.180(0.615-2.264)$ \\
\hline
\end{tabular}

common concurrent pathogens for pneumonia, and urinary tract infection was the most common concurrent infection. However, more patients had Serratia marcescnes coinfection and invasive fungal infection in tigecycline group, and more patients had Stenotrophomonas maltophilia coinfection and catheter related infection in sulbactam group. Among the 168 enrolled patients, bacteremia during treatment was observed in four patients, and all of them were from tigecycline group $(P=$ 0.121). During treatment, tigecycline-resistant MDR Acb complex was isolated in $16(19.0 \%)$ tigecycline-treated patients, and sulbactam-resistant MDR Acb complex was isolated in $43(51.2 \%)$ sulbactam or ampicllin/sulbactam-treated patients $(19.0 \%$ versus $51.2 \%, P<0.0001)$ (Table 1$)$. In tigecycline group, 71 patients $(84.5 \%)$ had airway MDR Acb complex isolates with full or intermediate resistance to sulbactam. Tigecycline susceptibility testing was not performed in sulbactam group.

\section{Treatment}

The mean treatment duration was 14.6 and 16.4 days for tigecycline and sulbactam group, respectively. Compared to the tigecycline group, more patients in sulbactam group had combination therapy (84.5\% versus $53.6 \%)$, particularly with carbapenems (71.4\% versus $6.0 \%)$. In sulbactam group, the most common co-administered agent were carbapenems (60/71, $84.5 \%)$, followed by cephalosporins $(8 / 71,11.3 \%)$, and 32 patients (38.1\%) had glycopeptides use. In tigecycline group, the most common co-administered agent was cephalosporins (20/ $45,44.4 \%)$, followed by colistin $(12 / 45,26.7 \%)$ and fluoroquinolones $(12 / 45,26.7 \%)$. Colistin was not available until May 2007 in our hospital, and co-use of colistin was only noted in tigecycline group. More patients in tigecycline group had delayed treatment (41.7\% versus $26.2 \%$ ) (Table 1 ).

\section{Outcomes}

Sulbactam group had a higher rate of airway MDR Acb complex eradication (63.5\% versus $33.3 \%$ ) and defervescence (90.5\% versus $64.3 \%$ ) than tigecycline group at the end of treatment. There was no significant difference between these two groups in the rates of clinical resolution, 30-day mortality and mortality during treatment (66.7\% versus $66.7 \%$; $33.3 \%$ versus $29.8 \%$; $25.0 \%$ versus $17.9 \%$, respectively) (Table 1 ). The cumulative survival rate at 30 days was similar between the two groups by Kaplan-Meier method (Fig. 1).

\section{The predictor for mortality during treatment}

In the univariate analysis, the survivors were more likely to have tigecycline or sulbactam-resistant MDR Acb complex than the deceased (39.4\% versus $19.4 \%)$ (Table 2). And most of the resistant isolates in survivors were from sulbactam group (39/52, $75 \%)$. In the multivariate analysis including tigecycline-based treatment and variables with a $P$ value $<0.1$ in the univariate analysis, bilateral pneumonia was the only independent predictor for mortality during treatment (adjusted OR, 2.717; $95 \%$ CI, 1.015 to 7.272) (Table 2). Other models of multivariate analysis including polymicrobial pneumonia, combination therapy, and combination with carbapenem or colistin also showed that bilateral pneumonia was the only independent predictor (Table 3). 


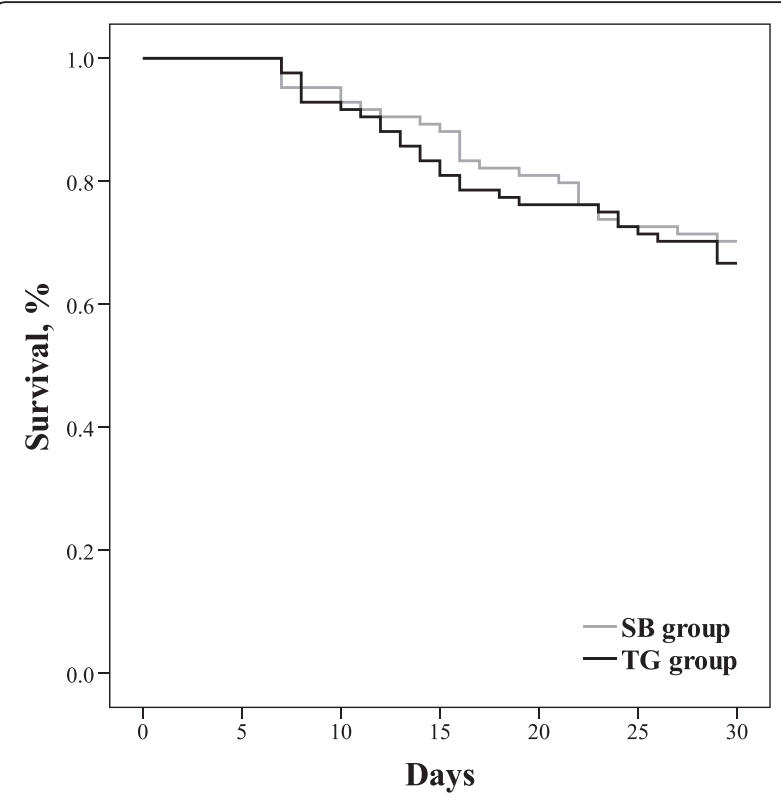

Fig. 1 Comparative survival curves for tigecycline (black line) and sulbactam (gray line) groups; Log-rank test: $p=0.605$. Abbreviations: SB sulbactam, TG tigecycline

\section{Monotherapy of tigecycline and sulbactam}

Thirty-nine (46.4\%) patients had tigecycline monotherapy and 13 (15.5\%) had sulbactam or ampicillin/sulbactam monotherapy. Tigecycline group had significant lower rates of ventilator use, bilateral pneumonia, and airway eradication of MDR Acb complex. Both groups had similar clinical resolution rates. However, tigecycline group had lower rates of 30-day mortality and mortality during treatment (25.6\% versus $53.8 \%, 17.9 \%$ versus $30.8 \%, P>0.05)$. In the univariate analysis for the patients with monotherapy, both the survivors and the deceased during treatment had no significant difference in demography, concomitant diseases, clinical conditions, and treatment (Table 4).

\section{Discussion}

Prior case series studies reported clinical response rates ranging from 60 to $88 \%$ in tigecycline treatment [9-11], and 67 to $75 \%$ in sulbactam or ampicillin/sulbactam treatment for pneumonia involving MDRAB or MDR Acb complex [13-15]. There were only a few comparative studies investigating the efficacy of tigecycline or sulbactam, and usually they were compared with colistin or polymyxin, the other major treatment option for MDR Acb complex. Betrosian AP et al. reported that high-dose ampicillin/sulbactam monotherapy and colistin were comparably safe and effective treatment for critically ill patients with MDRAB VAP. The clinical success and improvement rate was $76.9 \%$ for ampcillin/sulbactam group and $73.3 \%$ for colistin group [22]. Oliveira
MS et al. reported another study comparing ampicillin/ sulbactam with polymyxins in treating infections caused by carbapenem-resistant Acinetobacter spp. [23]. In the study, about half of the enrolled patients had Acinetobacter bacteremia, and quarter of them had pneumonia. The mortality rate during treatment was $33 \%$ in ampicillin/sulbactam group and $50 \%$ in polymyxin group, and polymyxin use was an independent factor associated with mortality during treatment [23]. Chuang YC et al. reported a study comparing tigecycline-based to colistinbased therapy for MDRAB pneumonia in intensive care units. The tigecycline group has an excess mortality of $16.7 \%(60.7 \%$ versus $44 \%, P=0.04)$. The excess mortality of tigecycline is significant only among those with minimal inhibitory concentration (MIC) $>2 \mu \mathrm{g} / \mathrm{mL}$, but not for those with $\mathrm{MIC} \leqq 2 \mu \mathrm{g} / \mathrm{mL}$ [24].

To our knowledge, the study was the first comparative study of tigecycline-based versus sulbactam or ampicillin/sulbactam-based treatment for pneumonia involving MDR Acb complex. Our two patient groups were from different but successive time periods, and the major treatment for MDR Acb complex was different in each time period in our hospital. Before August 2007, sulbactam or ampicillin/sulbactam was the only option probably with in vitro activity against MDR Acb complex in our hospital. After that, tigecycline became the major treatment option because of its high susceptibility rate to MDR Acb complex. However, the clinical and microbiological diagnostic criteria and definition, and standards of care and infection control were similar in both time periods. Covariate adjustment with multivariate analyses and matching with disease severity were performed to reduce bias of the historically controlled comparison.

The patients from both groups were aged with complicated underlying diseases and high disease severity. Higher rates of ventilator use and bilateral pneumonia reflected that sulbactam group might have a higher severity of pneumonia than tigecycline group. A higher rate of delay use in tigecycline group might reflect the early policy of tigecycline use in our hospital: usually tigecycline was not used as empiric or first-line regimen for nosocomial infection. Both groups had similar clinical outcomes. Bilateral pneumonia was the only independent predictor for mortality during treatment in different models of multivariate analysis. Combination therapy did not stand out as an independent predictor, which might be due to difference of combination strategies and regimens between the two patient groups. Most patients in sulbactam group had combination with carbapenem for synergistic effect against MDR Acb complex; however, tigecycline group mainly had anti-pseudomonal cephalosporins and fluoquinolones to cover Pseudomonas aeruginosa. 
Table 2 Univariate and multivariate analyses of the predictors for mortality during treatment of tigecycline or sulbactam or ampicillin/ sulbactam for pneumonia involving multidrug resistant Acinetobacter calcoaceticus-Acinetobacter baumannii (MDR Acb) complex

\begin{tabular}{|c|c|c|c|c|c|}
\hline \multirow[t]{2}{*}{ Variables } & \multirow{2}{*}{$\begin{array}{l}\text { Deceased }^{\text {a }} \\
n=36\end{array}$} & \multirow{2}{*}{$\begin{array}{l}\text { Survivors }^{\text {a }} \\
n=132\end{array}$} & \multirow{2}{*}{$\begin{array}{l}\text { Univariate } \\
p\end{array}$} & \multicolumn{2}{|c|}{ Multivariate $^{b}$} \\
\hline & & & & $p$ & Adjusted OR (95\% Cl) \\
\hline \multicolumn{6}{|l|}{ Demographic parameters } \\
\hline Age, yr & $69.8(14.7)$ & $70.2(16.0)$ & 0.658 & & \\
\hline Male gender & $26(72.2)$ & $89(67.4)$ & 0.583 & & \\
\hline \multicolumn{6}{|l|}{ Concomitant diseases } \\
\hline Hepatic dysfunction & $6(16.7)$ & $12(9.1)$ & 0.224 & & \\
\hline Renal insufficiency & $15(41.7)$ & $49(37.1)$ & 0.619 & & \\
\hline Chronic pulmonary disease & $6(16.7)$ & $36(27.3)$ & 0.193 & & \\
\hline Heart disease & $6(16.7)$ & $14(10.6)$ & 0.383 & & \\
\hline Diabetes mellitus & $14(38.9)$ & $47(35.6)$ & 0.717 & & \\
\hline Immune compromise & $5(13.9)$ & $19(14.4)$ & 0.939 & & \\
\hline Malignancy & $11(30.6)$ & $24(18.2)$ & 0.105 & & \\
\hline Surgery & $11(30.6)$ & $26(19.7)$ & 0.163 & & \\
\hline \multicolumn{6}{|l|}{ Clinical conditions } \\
\hline APACHE ॥ Score & $20.3(7.3)$ & $20.1(5.8)$ & 0.858 & & \\
\hline Ventilator use & $26(72.2)$ & $107(81.1)$ & 0.247 & & \\
\hline Bilateral pneumonia & $30(83.3)$ & $88(66.7)$ & 0.053 & 0.047 & $2.717(1.015-7.272)$ \\
\hline Polymicrobial pneumonia & 29 (80.6) & $99(75.0)$ & 0.488 & & \\
\hline With MRSA & $10(27.8)$ & $50(37.9)$ & 0.262 & & \\
\hline With Pseudomonas aeruginosa & $14(38.9)$ & $42(31.8)$ & 0.425 & & \\
\hline With Klebsiella spp. & $6(16.7)$ & $11(8.3)$ & 0.207 & & \\
\hline With Serratia marcescens & $3(8.3)$ & $8(6.1)$ & 0.704 & & \\
\hline With Stenotrophomanas maltophilia & $6(16.7)$ & $15(11.4)$ & 0.400 & & \\
\hline Multisite infections & $18(50.0)$ & $50(37.9)$ & 0.189 & & \\
\hline With urinary tract infection & $8(22.2)$ & $24(18.2)$ & 0.584 & & \\
\hline With catheter related infection & $2(5.6)$ & $10(7.6)$ & 1.000 & & \\
\hline With skin and soft tissue infection & $6(16.7)$ & $9(6.8)$ & 0.094 & 0.214 & $2.070(0.657-6.521)$ \\
\hline With intra-abdominal infection & $3(8.3)$ & $9(6.8)$ & 0.721 & & \\
\hline With invasive fungal infection & $3(8.3)$ & $13(9.8)$ & 1.000 & & \\
\hline \multicolumn{6}{|l|}{ Microbiology } \\
\hline MDR Acb complex with TG or SB resistance ${ }^{c}$ & $7(19.4)$ & $52(39.4)$ & 0.026 & 0.079 & $0.426(0.164-1.103)$ \\
\hline Airway eradication of MDR Acb complex ${ }^{d}$ & $11(42.3)$ & $62(49.2)$ & 0.521 & & \\
\hline Bacteremia during treatment & $2(5.6)$ & $2(1.5)$ & 0.201 & & \\
\hline \multicolumn{6}{|l|}{ Treatment } \\
\hline Tigecycline-based treatment & $21(58.3)$ & $63(47.7)$ & 0.259 & 0.451 & $1.371(0.604-3.116)$ \\
\hline Duration, days & $15.6(7.9)$ & $15.5(6.2)$ & 0.519 & & \\
\hline Combination therapy & $25(69.4)$ & $91(68.9)$ & 0.954 & & \\
\hline With cephalosporins & $6(16.7)$ & $22(16.7)$ & 1.000 & & \\
\hline With carbapenems & $12(33.3)$ & $53(40.2)$ & 0.457 & & \\
\hline With fluoroquinolones & $2(5.6)$ & $14(10.6)$ & 0.527 & & \\
\hline With colistin & $3(8.3)$ & $9(6.8)$ & 0.721 & & \\
\hline Delayed treatment & $9(25.0)$ & $48(36.4)$ & 0.202 & & \\
\hline
\end{tabular}

Abbreviations: MDR Acb multidrug resistant Acinetobacter calcoaceticus-Acinetobacter baumannii, OR odd ratio, $\mathrm{Cl}$ confidence interval, $A P A C H E$ acute physiology and chronic health evaluation, MRSA methicillin resistant Staphylococcus aureus, TG tigecycline, SB sulbactam

${ }^{a}$ Categorical data are no. (\%) of subject, continuous data are expressed as mean (standard deviation)

${ }^{\mathrm{b}}$ All variables included in the final multivariable model are shown

' The initial airway MDR Acb complex isolates with resistance to TG in TG group, or with resistance to SB in SB group

${ }^{d} 26$ patients in the deceased group and 126 in the survivors group had available data for evaluation 
Table 3 Multivariate analyses of the predictors for mortality during treatment including combination therapy, carbapenems or colistin use, and polymicrobial pneumonia

\begin{tabular}{|c|c|c|c|}
\hline Variables & Odds ratio & $95 \%$ confidence interval & $p$ \\
\hline \multicolumn{4}{|l|}{ Model $A^{a}$} \\
\hline With skin and soft tissue infection & 2.041 & $0.644-6.466$ & 0.225 \\
\hline MDR Acb complex with TG or SB resistance & 0.418 & $0.160-1.092$ & 0.075 \\
\hline Bilateral pneumonia & 2.663 & $0.987-7.186$ & 0.053 \\
\hline Tigecycline-based treatment & 1.405 & $0.608-3.245$ & 0.426 \\
\hline Combination therapy & 1.133 & $0.472-2.720$ & 0.779 \\
\hline \multicolumn{4}{|l|}{ Model $B^{b}$} \\
\hline With skin and soft tissue infection & 2.071 & $0.657-6.523$ & 0.214 \\
\hline MDR Acb complex with TG or SB resistance & 0.426 & $0.163-1.114$ & 0.082 \\
\hline Bilateral pneumonia & 2.717 & $1.007-7.329$ & 0.048 \\
\hline Tigecycline-based treatment & 1.373 & $0.497-3.795$ & 0.541 \\
\hline Combination with carbapenem & 1.002 & $0.346-2.905$ & 0.997 \\
\hline \multicolumn{4}{|l|}{ Model $C^{c}$} \\
\hline With skin and soft tissue infection & 2.002 & $0.624-6.425$ & 0.243 \\
\hline MDR Acb complex with TG or SB resistance & 0.420 & $0.161-1.090$ & 0.075 \\
\hline Bilateral pneumonia & 2.795 & $1.028-7.600$ & 0.044 \\
\hline Tigecycline-based treatment & 1.430 & $0.608-3.363$ & 0.413 \\
\hline Combination with colistin & 0.783 & $0.178-3.444$ & 0.746 \\
\hline \multicolumn{4}{|l|}{ Model D } \\
\hline With skin and soft tissue infection & 2.035 & $0.639-6.485$ & 0.230 \\
\hline MDR Acb complex with TG or SB resistance & 0.428 & $0.165-1.111$ & 0.081 \\
\hline Bilateral pneumonia & 2.711 & $1.013-7.254$ & 0.047 \\
\hline Tigecycline-based treatment & 1.362 & $0.598-3.102$ & 0.462 \\
\hline Polymicrobial pneumonia & 1.109 & $0.426-2.884$ & 0.833 \\
\hline \multicolumn{4}{|l|}{ Model E } \\
\hline With skin and soft tissue infection & 1.979 & $0.612-6.405$ & 0.254 \\
\hline MDR Acb complex with TG or SB resistance & 0.422 & $0.160-1.110$ & 0.080 \\
\hline Bilateral pneumonia & 2.781 & $1.014-7.624$ & 0.047 \\
\hline Tigecycline-based treatment & 1.422 & $0.498-4.056$ & 0.510 \\
\hline Polymicrobial pneumonia & 1.086 & $0.413-2.853$ & 0.868 \\
\hline Combination with colistin & 0.797 & $0.179-3.557$ & 0.767 \\
\hline Combination with carbapenem & 1.006 & $0.346-2.921$ & 0.991 \\
\hline
\end{tabular}

Abbreviations: MDR Acb multidrug resistant Acinetobacter calcoaceticus-Acinetobacter baumannii, TG tigecycline, SB sulbactam

${ }^{a}$ No significant predictor was revealed when model $A$ included polymicrobial pneumonia

${ }^{\mathrm{b}}$ Bilateral pneumonia was the only significant predictor when model B included polymicrobial pneumonia ( $p=0.049$, adjusted odds ratio, 2.709 ; $95 \%$ confidential interval, 1.004-7.305)

${ }^{c}$ Bilateral pneumonia was the only significant predictor when model C included polymicrobial pneumonia ( $p=0.045$, adjusted odds ratio, 2.783 ; $95 \%$ confidential interval, 1.023-7.569)

Because most patients in sulbactam group had concurrent carbapenem use, the study results in them might mainly reflect the efficacy of combination of sulbactam and carbapenem. In the comparative analyses of monotherapy, the patients with sulbactam or ampicillin/sulbactam monotherapy had relatively higher mortality rates than the patients with tigecycline monotherapy or the overall sulbactam group. The results implied that combination with carbapenem might improve clinical outcomes of sulbactam-based treatment. Besides, more than half of the sulbactam group had sulbactam-resistant MDR Acb complex isolates during treatment. Combination with carbapenem might play a role giving a high airway eradication rate. The 
Table 4 The comparison and outcome analyses of the patients with monotherapy of tigecycline or sulbactam

\begin{tabular}{|c|c|c|c|c|c|c|}
\hline \multirow[t]{2}{*}{ Variables } & TG group ${ }^{\mathrm{a}}$ & SB group ${ }^{a}$ & $p$ & \multirow{2}{*}{$\begin{array}{l}\text { Deceased }^{\text {ab }} \\
n=11\end{array}$} & Survivors $^{\text {a }}$ & \multirow[t]{2}{*}{$p$} \\
\hline & $n=39$ & \multicolumn{2}{|l|}{$n=13$} & & $n=41$ & \\
\hline \multicolumn{7}{|l|}{ Demographic parameters } \\
\hline Age, yr & $71.4(15.0)$ & $68.7(19.9)$ & 0.899 & $75.3(12.0)$ & $69.5(17.1)$ & 0.439 \\
\hline Male gender & $25(64.1)$ & $10(76.9)$ & 0.506 & $8(72.7)$ & $27(65.9)$ & 1.000 \\
\hline \multicolumn{7}{|l|}{ Concomitant diseases } \\
\hline Hepatic dysfunction & $3(7.7)$ & $0(0.0)$ & 0.564 & $1(9.1)$ & $2(4.9)$ & 0.518 \\
\hline Renal insufficiency & $10(25.6)$ & $4(30.8)$ & 0.729 & $1(9.1)$ & $13(31.7)$ & 0.251 \\
\hline Chronic pulmonary disease & $11(28.2)$ & $4(30.8)$ & 1.000 & $4(36.4)$ & $11(26.8)$ & 0.709 \\
\hline Heart disease & $6(15.4)$ & $1(7.7)$ & 0.664 & $2(18.2)$ & $5(12.2)$ & 0.630 \\
\hline Diabetes mellitus & $9(23.1)$ & $4(30.8)$ & 0.714 & $3(27.3)$ & $10(24.4)$ & 1.000 \\
\hline Immune compromise & $8(20.5)$ & $1(7.7)$ & 0.420 & $2(18.2)$ & $7(17.1)$ & 1.000 \\
\hline Malignancy & $8(20.5)$ & $3(23.1)$ & 1.000 & $4(36.4)$ & $7(17.1)$ & 0.216 \\
\hline Surgery & $9(23.1)$ & $1(7.7)$ & 0.419 & $2(18.2)$ & $8(19.5)$ & 1.000 \\
\hline \multicolumn{7}{|l|}{ Clinical conditions } \\
\hline APACHE II Score & $17.0(6.1)$ & $18.2(6.0)$ & 0.557 & $17.1(6.8)$ & $17.3(5.9)$ & 0.904 \\
\hline Ventilator use & $21(53.8)$ & $11(84.6)$ & 0.048 & $6(54.5)$ & $26(63.4)$ & 0.730 \\
\hline Bilateral pneumonia & $18(46.2)$ & $12(92.3)$ & 0.004 & $8(72.7)$ & $22(53.7)$ & 0.319 \\
\hline Polymicrobial pneumonia & $31(79.5)$ & $9(69.2)$ & 0.466 & $9(81.8)$ & $31(75.6)$ & 1.000 \\
\hline With MRSA & $20(51.3)$ & $6(46.2)$ & 0.749 & $5(45.5)$ & $21(51.2)$ & 0.734 \\
\hline With Pseudomonas aeruginosa & $14(35.9)$ & $3(23.1)$ & 0.506 & $5(45.5)$ & $12(29.3)$ & 0.470 \\
\hline With Klebsiella spp. & $7(17.9)$ & $1(7.7)$ & 0.662 & $1(9.1)$ & $7(17.1)$ & 1.000 \\
\hline With Serratia marcescens & $5(12.8)$ & $1(7.7)$ & 1.000 & $1(9.1)$ & $5(12.2)$ & 1.000 \\
\hline With Stenotrophomanas maltophilia & $3(7.7)$ & $3(23.1)$ & 0.157 & $1(9.1)$ & $5(12.2)$ & 1.000 \\
\hline Multisite infections & $14(35.9)$ & $5(38.5)$ & 1.000 & $4(36.4)$ & $15(36.6)$ & 1.000 \\
\hline With urinary tract infection & $7(17.9)$ & $3(23.1)$ & 0.697 & $2(18.2)$ & $8(19.5)$ & 1.000 \\
\hline With catheter related infection & $0(0.0)$ & $2(15.4)$ & 0.059 & $1(9.1)$ & $1(2.4)$ & 0.382 \\
\hline With skin and soft tissue infection & $3(7.7)$ & $1(7.7)$ & 1.000 & $1(9.1)$ & $3(7.3)$ & 1.000 \\
\hline With intra-abdominal infection & $4(10.3)$ & $0(0.0)$ & 0.561 & $0(0.0)$ & $4(9.8)$ & 0.567 \\
\hline With invasive fungal infection & $6(15.4)$ & $0(0.0)$ & 0.317 & $1(9.1)$ & $5(12.2)$ & 1.000 \\
\hline \multicolumn{7}{|l|}{ Microbiology } \\
\hline MDR Acb complex with TG or SB resistance ${ }^{c}$ & $6(15.4)$ & $4(30.8)$ & 0.244 & $1(9.1)$ & $9(22.0)$ & 0.668 \\
\hline Airway eradication of MDR Acb complex ${ }^{d}$ & $12(34.3)$ & $8(88.9)$ & 0.006 & $4(57.1)$ & $16(43.2)$ & 0.684 \\
\hline Bacteremia during treatment & $2(5.1)$ & $0(0.0)$ & 1.000 & $1(9.1)$ & $1(2.4)$ & 0.382 \\
\hline \multicolumn{7}{|l|}{ Treatment } \\
\hline Tigecycline-based treatment & & & & $7(63.6)$ & $32(78.0)$ & 0.435 \\
\hline Duration, days & $13.8(5.1)$ & $12.7(5.6)$ & 0.293 & $11.9(3.0)$ & $14.0(5.6)$ & 0.398 \\
\hline Delayed treatment & $20(51.3)$ & $5(38.5)$ & 0.423 & $5(45.5)$ & $20(48.8)$ & 0.845 \\
\hline \multicolumn{7}{|l|}{ Outcomes } \\
\hline Clinical resolution of pneumonia & $26(66.7)$ & $8(61.5)$ & 0.747 & & & \\
\hline Mortality during treatment & $7(17.9)$ & $4(30.8)$ & 0.435 & & & \\
\hline 30-day mortality & $10(25.6)$ & $7(53.8)$ & 0.089 & & & \\
\hline
\end{tabular}

Abbreviations: TG tigecycline, SB sulbactam, APACHE acute physiology and chronic health evaluation, MRSA methicillin resistant Staphylococcus aureus, MDR Acb multidrug resistant Acinetobacter calcoaceticus-Acinetobacter baumannii

${ }^{a}$ Categorical data are no.(\%) of subject, continuous data are expressed as mean (standard deviation)

${ }^{\mathrm{b}}$ Mortality during treatment

'The initial airway MDR Acb complex isolates with resistance to TG in TG group, or with resistance to SB in SB group

$\mathrm{d}_{35}$ patients in TG group and 9 in SB group; 7 patients in the deceased group and 37 in the survivors group had available data for evaluation 
patients with sulbactam monotherapy also had a high airway eradication rate, but most of them did not have sulbactam-resistant MDR Acb complex.

Synergistic effect against MDRAB with the combination of sulbactam and carbapenem had been reported $[4,25]$. However, the synergistic effect was associated with the MICs of carbapenem and sulbactam. If the MICs exceeded achievable serum levels, the potential of sulbactam/carbapenem combination as treatment regimen for MDRAB infections might be limited [4]. In our study, full or intermediate sulbactam resistance was detected in $84.5 \%$ of tigecycline-treated patients, therefore, physicians tended to use tigecycline for these patients with sulbactam-resistant MDB Acb complex. For these cases, the clinical outcomes of sulbactam group might not be achieved if they received sulbactam/carbapenem combination therapy.

There are some other limitations in this study. First, our respiratory specimens were clinical specimens from clinical practice, and they might not be obtained from deep sites in the lungs. Growths of etiologic pathogens were assessed semi-quantitatively if the specimens were qualified for culture. We cannot absolutely distinguish airway MDR Acb complex infections from colonization. However, our definition for pneumonia was practical, and our conclusion based on clinically relevant data and management could provide important information for clinical practice. Second, polymicrobial pneumonia and concomitant infections were common, and the clinical impact of other etiologic pathogens or extrapulmonary infections was not evaluated comprehensively. Third, we studied MDR Acb complex rather than MDRAB. Although prior studies reported that about $90 \%$ of Acb complex with multidrug or carbapenem resistance was the genomic specie of A.baumannii, comparison with studies on $A$. baumannii isolates are not straightforward [26].

\section{Conclusions}

Tigecycline-based treatment had a similar clinical outcome to sulbactam or ampicillin/sulbactam-based treatment for pneumonia involving MDR Acb commplex, but tigecycline group had a lower microbiological eradiation rate. More comparison studies are essential to establish the optimal regimens for pneumonia involving MDR Acb complex.

\footnotetext{
Abbreviations

AB, Acinetobacter baumannii; Acb, Acinetobacter calcoaceticus-Acinetobacter baumannii; APACHE, acute physiology and chronic health evaluation; $\mathrm{CGMH}$, Chang Gung Memorial Hospital; Cl, confidence interval; MDR, multidrugresistant; MIC, minimal inhibitory concentration; OR, odds ratio; VAP, ventilator associated pneumonia
}

\section{Acknowledgments}

This retrospective study has been approved by institutional review boards of CGMH- Linkou (Number: 99-1478B and 100-0294B). The ethics committee granted a waiver for informed consent to be obtained. No funding sources had any role in the design or conduct of the study; collection, management, analysis, or interpretation of the data; or preparation, review, or approval of the manuscript.

\section{Authors' contributions}

$J J Y$, and HSL reviewed the medical records and drafted the manuscript; CFY, YMW, PYH, and CCY analyzed and interpreted the data. JJY, MHL, and CTH designed and oversaw the study, analyzed and interpreted the data, and revised the manuscript. All authors have read and approved the manuscript for publication.

\section{Competing interests}

The authors declare that they have no competing interests.

\section{Author details}

'Division of Infectious Diseases, Department of Internal Medicine, Chang Gung Memorial Hospital at Linkou, Chang Gung University College of Medicine, 5 Fu-Shin St.Gueishan 333 Taoyuan, Taiwan. ²Division of Infectious Diseases, Department of Internal Medicine, Chang Gung Memorial Hospital, Cha-Yi, Taiwan.

Received: 30 March 2015 Accepted: 14 July 2016

Published online: 05 August 2016

\section{References}

1. Playford EG, Craig JC, Iredell JR. Carbapenem-resistant Acinetobacter baumannii in intensive care unit patients: risk factors for acquisition, infection and their consequences. J Hosp Infect. 2007;65(3):204-11.

2. Sunenshine $R H$, Wright MO, Maragakis LL, Harris AD, Song X, Hebden J, Cosgrove SE, Anderson A, Carnell J, Jernigan DB, Kleinbaum DG, Perl TM, Standiford HC, Srinivasan A. Multidrug-resistant Acinetobacter infection mortality rate and length of hospitalization. Emerg Infect Dis. 2007;13(1):97-103.

3. Ye JJ, Huang CT, Shie SS, Huang PY, Su LH, Chiu CH, Leu HS, Chiang PC. Multidrug resistant Acinetobacter baumannii: risk factors for appearance of imipenem resistant strains on patients formerly with susceptible strains. PLoS One. 2010;5(4):e9947. doi:10.1371/journal.pone.0009947.

4. Hsueh PR, Teng LJ, Chen CY, Chen WH, Yu CJ, Ho SW, Luh KT. Pandrugresistant Acinetobacter baumannii causing nosocomial infections in a university hospital, Taiwan. Emerg Infect Dis. 2002;8(8):827-32.

5. Giamarellou H, Poulakou G. Multidrug-resistant Gram-negative infections: what are the treatment options? Drugs. 2009;69(14):1879-901.

6. Hoban DJ, Bouchillon SK, Johnson BM, Johnson JL, Dowzicky MJ, Tigecycline Evaluation and Surveillance Trial (TEST Program) Group. In vitro activity of tigecycline against 6,792 Gram-negative and Gram-positive clinical isolates from the global Tigecycline Evaluation and Surveillance Trial (TEST Program; 2004). Diagn Microbiol Infect Dis. 2005;52(3):215-27.

7. FDA Drug Safety Communication. Increased risk of death with Tygacil (tigecycline) compared to other antibiotics used to treat similar infections. Available at: http://www.fda.gov/Drugs/DrugSafety/ucm224370.htm. Accessed Sept 2010.

8. Freire AT, Melnyk V, Kim MJ, Datsenko O, Dzyublik O, Glumcher F, Chuang YC, Maroko RT, Dukart G, Cooper CA, Korth-Bradley JM, Dartois N, Gandjini H, 311 Study Group. Comparison of tigecycline with imipenem/cilastatin for the treatment of hospital-acquired pneumonia. Diagn Microbiol Infect Dis. 2010;68(2):140-51.

9. Curcio D, Fernández F, Vergara J, Vazquez W, Luna CM. Late onset ventilator-associated pneumonia due to multidrug-resistant Acinetobacter spp.: experience with tigecycline. J Chemother. 2009;21(1):58-62.

10. Poulakou G, Kontopidou FV, Paramythiotou E, Kompoti M, Katsiari M, Mainas E, Nicolaou C, Yphantis D, Antoniadou A, Trikka-Graphakos E, Roussou Z, Clouva P, Maguina N, Kanellakopoulou K, Armaganidis A, Giamarellou H. Tigecycline in the treatment of infections from multi-drug resistant gramnegative pathogens. J Infect. 2009;58(4):273-84.

11. Ye JJ, Lin HS, Kuo AJ, Leu HS, Chiang PC, Huang CT, Lee MH. The clinical implication and prognostic predictors of tigecycline treatment for pneumonia involving multidrug-resistant Acinetobacter baumannii. J Infect. 2011;63(5):351-61.

12. Corbella X, Ariza J, Ardanuy C, Vuelta M, Tubau F, Sora M, Pujol M, Gudiol F. Efficacy of sulbactam alone and in combination with ampicillin in nosocomial infections caused by multiresistant Acinetobacter baumannii. J Antimicrob Chemother. 1998;42(6):793-802. 
13. Betrosian AP, Frantzeskaki F, Xanthaki A, Georgiadis G. High-dose ampicillinsulbactam as an alternative treatment of late-onset VAP from multidrugresistant Acinetobacter baumannii. Scand J Infect Dis. 2007;39(1):38-43.

14. Levin AS, Levy CE, Manrique AE, Medeiros EA, Costa SF. Severe nosocomial infections with imipenem-resistant Acinetobacter baumannii treated with ampicillin/sulbactam. Int J Antimicrob Agents. 2003;21(1):58-62.

15. Lin HS, Lee MH, Cheng CW, Hsu PC, Leu HS, Huang CT, Ye JJ. Sulbactam treatment for pneumonia involving multidrug-resistant Acinetobacter calcoaceticus-Acinetobacter baumannii complex. Infect Dis. 2015;47(6):370-8.

16. American Thoracic Society, Infectious Diseases Society of America. Guidelines for the management of adults with hospital-acquired, ventilatorassociated, and healthcare-associated pneumonia. Am J Respir Crit Care Med. 2005;171(4):388-416.

17. Fujitani S, Cohen-Melamed MH, Tuttle RP, Delgado E, Taira Y, Darby JM. Comparison of semi-quantitative endotracheal aspirates to quantitative non-bronchoscopic bronchoalveolar lavage in diagnosing ventilatorassociated pneumonia. Respir Care. 2009;54(11):1446-8.

18. Schreckenberger PC, Daneshvar MI, Weyant RS, Hollis DG. Acinetobacter, Achromobacter, Chryseobacerium, Moraxella and other nonformentative gram-negative rods. In: Versalovic J, Carroll KC, Funke G, Jorgensen JH, Louise-Landry M, Warnock DW, editors. Manual of clinical microbiology. 10th ed. Washington: American Society of Microbiology; 2011. p. 714-38.

19. Clinical and Laboratory Standards Institute. Performance standards for antimicrobial susceptibility testing: twenty-first informational supplement M100-S21. Wayne: CLSI; 2011

20. Jones RN, Ferraro MJ, Reller LB, Schreckenberger PC, Swenson JM, Sader HS Multicenter studies of tigecycline disk diffusion susceptibility results for Acinetobacter spp. J Clin Microbiol. 2007;45(1):227-30.

21. Paterson DL. The Epidemiological profile of infections with multidrugresistant Pseudomonas aeruginosa and Acinetobacter species. Clin Infect Dis. 2006;43(S2):S43-48.

22. Betrosian AP, Frantzeskaki F, Xanthaki A, Douzinas EE. Efficacy and safety of high-dose ampicillin/sulbactam vs. colistin as monotherapy for the treatment of multidrug resistant Acinetobacter baumannii ventilatorassociated pneumonia. J Infect. 2008:56(6):432-6.

23. Oliveira MS, Prado GV, Costa SF, Grinbaum RS, Levin AS. Ampicillin/ sulbactam compared with polymyxins for the treatment of infections caused by carbapenem-resistant Acinetobacter spp. J Antimicrob Chemother. 2008:61 (6):1369-75

24. Chuang YC, Cheng CY, Sheng WH, Sun HY, Wang JT, Chen YC, Chang SC. Effectiveness of tigecycline-based versus colistin- based therapy for treatment of pneumonia caused by multidrug-resistant Acinetobacter baumannii in a critical setting: a matched cohort analysis. BMC Infect Dis. 2014:14:102. doi:10.1186/1471-2334-14-102.

25. Lee NY, Wang CL, Chuang YC, Yu WL, Lee HC, Chang CM, Wang LR, Ko WC. Combination carbapenem-sulbactam therapy for critically ill patients with multidrug-resistant Acinetobacter baumannii bacteremia: four case reports and an in vitro combination synergy study. Pharmacotherapy. 2007:27(11):1506-11.

26. Lee YC, Huang YT, Tan CK, Kuo YW, Liao CH, Lee PI, Hsueh PR. Acinetobacter baumannii and Acinetobacter genospecies $13 \mathrm{TU}$ and 3 bacteraemia: comparison of clinical features, prognostic factors and outcomes. J Antimicrob Chemother. 2011;66(8):1839-46.

\section{Submit your next manuscript to BioMed Central and we will help you at every step:}

- We accept pre-submission inquiries

- Our selector tool helps you to find the most relevant journal

- We provide round the clock customer support

- Convenient online submission

- Thorough peer review

- Inclusion in PubMed and all major indexing services

- Maximum visibility for your research

Submit your manuscript at www.biomedcentral.com/submit

) Biomed Central 